\title{
Displacement transformation in gravitational wave detector
}

\section{Krysanov V.A.}

Institute for Nuclear Research of RAS, Lomonosov Moscow State University;

E-mail: Krysanov<kv@sai.msu.ru>;

In the full-scale OGRAN detector transfer of oscillatory displacements from end surfaces of the acoustic resonator to the Fabry-Perot cavity mirrors is considered. The elasticity of a mirror fastening element is taken into account. The mode in which mirror oscillation amplitude exceeds end surface amplitude is considered. Influence of the additional Boltzmann force is investigated. The influence of displacement measurer resolution to detector sensitivity is regarded. Phenomenological algorithm of data transformation is described; it realizes transformation of stochastic displacement spectral density into spectral density of metrics variations. A "reception band-width at the metric noise control level" is interpreted as a new characteristic. The corresponding characteristics of the GW detector "Ulitka" are calculated by the algorithm for comparison. The base analytic metric sensitivity algorithm using noise factor $F$ is considered and modifications are made in it. It is generalized on area $\mathrm{F} \leq 10$; it allows to exclude forecasting values $F<1$ and to determine a band of frequencies in which the detector potential sensitivity is realized $(F \leq 2)$. Noise factor expression is changed for taking into account a Pound-Drever-Hall technique and a FM discriminator. In addition to photoelectron shot noise contributions of laser radiation frequency and power fluctuations are presented.

Keywords: laser, cavity, noise, fluctuations, resolution, spectrum, metric, sensitivity, bandwidth.

DOI: $10.18698 / 2309-7604-2015-1-230-247$

\section{Introduction}

The basis of a GW bar detector is a massive cylindrical acoustic resonator [1]. To register small resonator length variations the mirrors of Fabry-Perot (FP) optical cavity are fastened to the bar ends [2]. In the OGRAN project the designed displacement resolution is $10^{-16} \mathrm{~cm} / \mathrm{Hz}^{1 / 2}$ [3]; optoelectronic registration system in Pound-Drever-Hall (PDH) technique is realized [4].

The detector is developed by Sternberg astronomical institute (SAI) of the MSU and Institute of Laser Physics (ILP) of the RAS. ILP has created registration system of small cavity length variations. The system contains the laser with high-speed frequency tuning, optical and electronic partitions. This is a main and unique technological content of the OGRAN Project. In the technology aspect MSU provides the infrastructure, vacuum equipment, mechanical part and FP cavities. MSU performs the studying and the test of the OGRAN installation in assembly. MSU has established the theoretical basis of the GW detector sensitivity, had formulated the requirements to the parameters of laser displacement meter and performs the transformation of test spectrograms into detector threshold characteristics in gravitational field metric variations. 
Institute for Nuclear Researches (INR) of RAS provides the chamber and infrastructure in the mountain gallery of the Baksan Neutrino Observatory (BNO). It was assumed that INR will exploit the instrument after repetition of announced characteristics in BNO at the end of the transition period. Exclusive point of view to installation is defined by the accepting side role; the metrology questions and the equipment characteristics control is a main task.

The installation has been moved to BNO. Four reports summarize the results of the Moscow period of detector creation [5-8]. It became clear that INR is obliging to involvement in the achievements of ILP and SAI MSU [8]. It defines the special attention to installation parameters and characteristics whereas the representation of important details isn't aim of the articles. As is customary, the preliminary installation sensitivity analysis is denoted. However, this analysis allows us to make some additions and corrections in the applied radio physics aspect.

By the time of installation disassembly the development is at the intermediate stage in which setup noise characteristics are defined by means of the spectrum analyzer and of nontransparent "optical calibration". The modest spectrogram frequency resolution determines small time intervals of data accumulation, whereas signal and noise characteristics of the workable detector "Ulitka" are determined by computer processing of large data arrays [9].

In the first Weber bar detector perturbations of an acoustic resonator were registered by passive piezocheramic sensors [1]; similar sensor is used in the detector "Ulitka" [10]. Complicated active parametrical displacement measurers had been intensely developed in MSU. In those schemes the steepness of signal conversion is provided by high quality factor of radiofrequency tanks and cavities. In OGRAN project the transition from such circuits [11-13] to system using FP cavity having exclusively high quality factor [2] has carried out [14, 15].

The initial scheme [2] is a basis of GWD sensitivity analysis [14, 15]. In the case of optimal signal filtering, the minimum detectable metric perturbation $h_{\min }$ is determined as $[7,8]$

$$
\begin{aligned}
& \mathrm{h}_{\min } \approx(4 / L) \sqrt{\left(k_{B} T / M \omega_{\mu}^{2}\right)\left(1 / \mathrm{Q} \omega_{\mu} \tau\right)} \approx(4 / L) \sqrt{\left(k_{B} T / M \omega_{\mu}^{3} \mathrm{Q}\right)_{\Delta} \mathrm{f}_{s}} \sqrt{F} \approx \\
& \approx 10^{-20} \sqrt{F \Delta f} H z^{-1 / 2} .
\end{aligned}
$$

Here $L$ is a bar length, $M$ is a equivalent mass, $\omega_{\mu}$ is a bar eigenfrequency, $Q$ is a bar quality factor, $\tau$ is duration of a special signal pulse, $\Delta f_{S} \approx(1 / \tau)$ is a frequency band, $F$ is a factor representing noise contribution of the laser displacement registration system.

The registration of the bar thermal motion as a narrow peak in the noise spectrogram has been the main recent achievement of the Project. The excess noise introduced by the mirror 
fastenings elements was identified and described in details [5]. The "background" of spectrum was lowered to level of $4 \cdot 10^{-15} \mathrm{~cm} / \mathrm{Hz}^{1 / 2}$. This resolution allows to definite general characteristics of the OGRAN installation in phenomenological consideration. Also it allows obtaining some information on mechanical parameters of mirror fastening elements without a dynamic test.

The OGRAN pilot model is composed. Sensitivity to the bar oscillations was declared at level of $(1 \div 2) \cdot 10^{-14} \mathrm{~cm} / \mathrm{Hz}^{1 / 2}$ [3]. Just the same value is presented in ref. [16]. In ref. [17] the measurer resolution of $10^{-14} \mathrm{~cm} / \mathrm{Hz}^{1 / 2}$ is declared. No peaks had been revealed in pilot model noise spectrogram [17], whereas the bar thermal peak is calculated as $7 \cdot 10^{-14} \mathrm{~cm} / \mathrm{Hz}^{1 / 2}[18,19]$.

Soon the sensitivity of the full-scale OGRAN setup achieve the of value $2 \cdot 10^{-15} \mathrm{~cm} / \mathrm{Hz}^{1 / 2}$ [6]. It is explained by minimization of the residual amplitude modulation (RAM) of laser radiation. This noise source is entered into consideration, described and estimated in ref. [20]; there is another reference [6]. The same "background" level one should see at fig. 6 in ref. [7] and at fig. 3 in ref. [8]; the articles indicate RAM noise too.

In ref. [18] the question of influence on detector sensitivity of phenomenological displacement measurer resolution was raised. The answer is contained in ref. [7, 8], where the displacement noise spectrogram is transformed into metrics variations spectrum. But the algorithm of transformation hasn't presented. It is represented below.

The rigorous analytical method of detector sensitivity forecasting is based by formula (1). This algorithm is not coordinated with phenomenological one. However, at engineering development of any device it is necessary to achieve rapprochement of theoretically predicted and measured values. On the way of the convergence the theoretical basis can be adapted.

\section{Detector potential sensitivity}

For analysis an acoustic resonator is reduced to an oscillator with equivalent parameters [2]

$$
M \ddot{x}+H_{\mu} \dot{X}+k_{\mu} X=F_{S}+F_{B},
$$

where $x$ is a mechanical displacement, $k_{\mu}$ is stiffness, $H_{\mu}$ is viscosity coefficient, $F_{S}$ is a signal force, $F_{B}$ is the stochastic Nyquist force; it is characterized by a power spectral density (PSD) $G_{B}$

$$
G_{B}(f)=4 k_{B} T H_{\mu}=4 k_{B} T M \omega_{\mu} / Q, \quad G_{B}(\omega)=2 k_{B} T M \omega_{\mu} / \pi Q .
$$


Here $\omega_{\mu}=\left(k_{\mu} / M\right)^{1 / 2}, Q=M \omega_{\mu} / H_{\mu}, \omega=2 \pi f$.

Gravitational field metric perturbations are reduced to an equivalent force [14, 21]

$$
F_{S m}=h_{m} M \omega_{\mu}^{2} L / 2
$$

where $L$ is a bar length, $h_{m}$ is a metric variation amplitude.

In accordance with a statistical theory, if variables have amplitude dependence as $y_{m}=K x_{m}$, where transfer parameter $K$ is a constant, the spectral densities of appropriate stochastic processes have relation $G_{y}=K^{2} G_{x}$. Then from expression (4) follows: $G_{F}=\left(M \omega_{\mu}{ }^{2} L / 2\right)^{2} G_{h}$. Assuming $G_{F}$ $=G_{B}$ one find formula for PSD of the detector threshold metric variations $G_{h}$

$$
G_{h}(f)=(4 / L)^{2} k_{B} T /\left(M Q \omega_{\mu}{ }^{3}\right)
$$

This formula coincides with the expressions (1) when $F=1$. A force registration method is not considered here. Small forces are determined by means of test body's displacements [2].

Consider the conditions of the potential sensitivity realization in a real instrument. Thermal Brownian motion of a bar registered by the displacement meter is described in ref. [19]. For harmonic signal $F_{S}(t)=F_{\mathrm{m}} \cos \omega \mathrm{t}$ from equation (2) around resonance $\left(\omega-\omega_{\mu} \ll \omega_{\mu}\right.$ the expres-sion for transfer parameter $K_{\mathrm{S}}$ is given as

$$
\left.K_{S}(\omega)=X_{m} / F_{m}=\left(Q / M \omega_{\mu}^{2}\right)\left[1+\left(\omega-\omega_{\mu}\right)^{2} / \delta_{\mu}\right)^{2}\right]^{-1 / 2},
$$

where $x_{m}$ is the amplitude of forced oscillating displacements, $\delta_{\mu}=\omega_{\mu} / 2 Q=H_{\mu} / 2 M$.

The PSD of thermal noise in the displacements $\mathrm{G}_{X B}$ as above is determined by the relation $\mathrm{G}_{X B}=\left|K_{\mathrm{S}}\right|^{2} G_{B}$. From the expressions (3) and (6) one find

$$
\begin{aligned}
G_{X B}(\omega) & \left.=\left(2 k T Q / \pi M \omega_{\mu}{ }^{3}\right)\left[1+\left(\omega-\omega_{\mu}\right)^{2} / \delta_{\mu}\right)^{2}\right]^{-1}= \\
& \left.=G_{X B}\left(\omega_{\mu}\right)\left[1+\left(\omega-\omega_{\mu}\right)^{2} / \delta_{\mu}\right)^{2}\right]^{-1} .
\end{aligned}
$$

The "background" input noise of the displacement meter $G_{S}(f)$ is added to this heat peak 


$$
\begin{gathered}
\left.G_{X}(f)=G_{X B}(f)+G_{S}(f)=G_{B 0} /\left[1+\left(\omega-\omega_{\mu}\right)^{2} / \delta_{\mu}\right)^{2}\right]+G_{S}, \\
G_{B 0} \equiv G_{X B}\left(f_{\mu}\right)=2 \pi G_{X B}\left(\omega_{\mu}\right)=4 k T Q / M \omega_{\mu}{ }^{3} .
\end{gathered}
$$

In the experimental installation OGRAN the dependence $\left[G_{X}(\omega)\right]^{1 / 2}$, obtained by means of the spectrum analyzer, is represented by figure 7 in ref. [7] and by figure 4 in ref. [8].

We begin presentation transformation of heat displacement spectrogram of into spectrogram of metric variations. First, using the expressions (4) and (6) we obtain

$$
\left.X_{m}=h_{m} Q(L / 2) /\left[1+\left(\omega-\omega_{\mu}\right)^{2} / \delta_{\mu}\right)^{2}\right]^{1 / 2} .
$$

As above, we can find the relation between the spectral densities of the corresponding stochastic processes. The signal transmission coefficient $K_{x h}$ has the view

$$
\left.K_{x h} \equiv X_{m} / h_{m}=Q(L / 2) /\left[1+\left(\omega-\omega_{\mu}\right)^{2} / \delta_{\mu}\right)^{2}\right]^{1 / 2}
$$

The next step of the phenomenological algorithm is determined by an expression

$$
\left(G_{h}\right)^{1 / 2}=\left(G_{X}\right)^{1 / 2} / K_{x h}
$$

It leads directly to the result reliance on figure 8 [7] (5 [8]) by analogy with the ref. [4].

We may emphasize the frequency range in which the potential sensitivity is realized. There are two frequencies $f_{H}$ and $f_{L}$, in which the condition $G_{X B}(f)=G_{S}$ is run. At these frequencies the total noise (8) is $3 \mathrm{~dB}$ higher than the background $G_{S}$. Within this area by means of expression (11) we obtain the expression (5), which have no frequency dependence.

Find the formulas for determining the potential sensitivity bandwidth (PSBW) $\Delta f_{B}=f_{H}-f_{L}$ . In the expression (7) for values of $\left|\omega-\omega_{\mu}\right|=\delta_{\mu}$ the level $-3 \mathrm{~dB}$ is realized and executes the ratio $\omega_{\mu} / 2 \delta_{\mu}=Q$. This is the standard resonant bandwidth $2 \delta_{\mu}$. For the reliance $\mathrm{G}_{X B}(f)$ we have the expressions for it's the standard bandwidth: $\Delta f_{0}=2 \delta_{\mu} / 2 \pi, \omega_{\mu}=2 \pi f_{\mu}, \Delta f_{0}=f_{\mu} / Q$.

When $\left|\omega-\omega_{\mu}\right| \geq 3 \delta_{\mu}$ the reliance (7) $\left(\Delta f=f-f_{\mu}\right)$ gets the simple view: 


$$
\left[G_{X B}(f)\right]^{1 / 2}=\left[G_{B 0}\right]^{1 / 2} \delta_{\mu} /\left|\omega-\omega_{\mu}\right|=\left[G_{B 0}\right]^{1 / 2}\left(\Delta f_{0} / 2\right) /|\Delta f|
$$

We introduce parameter $A_{S}$, determining from the experimental spectrogram (8)

$$
A_{S}=\left(G_{B 0}\right)^{1 / 2} /\left(G_{S}\right)^{1 / 2}
$$

Then from reliance (12) the relationship is following

$$
\Delta f_{B}=A_{S} \Delta f_{0}
$$

Consider characteristics of the experimental spectrogram. In the basic references $[2,14$, 15] mirrors are presumed to be fixed rigidly at the bar ends. Therefore cavity eigenfrequency variations $\delta v_{S}$ are connected with bar length variations $\delta x$ by the relation $\delta v_{S} / v=\delta x / L$ or

$$
d x=(I L / c) d n_{S}
$$

where $\lambda$ and $v$ are wavelength and optical frequency of laser infrared radiation.

This ratio provides a measurement of signal and noise variations of bar length through FP cavity. When $L=2 \mathrm{~m}$ and $\lambda=1,06 \cdot 10^{-4} \mathrm{~cm}$ the relation is $\delta \mathrm{x} / \delta v_{\mathrm{S}} \cong 0,7 \cdot 10^{-12} \mathrm{~cm} / \mathrm{Hz}$. It determines the ratio between the left and right ordinate scales of on the experimental spectrograms $[5,7,8]$.

As an important example, the background level $0,003 \mathrm{~Hz} / \mathrm{Hz}^{1 / 2}$ in fig. 6 and text [7] defines the measurer resolution of $\left(G_{S}\right)^{1 / 2} \cong 2 \cdot 10^{-15} \mathrm{~cm} / \mathrm{Hz}^{1 / 2}$; just this achievement is reported in ref. [6].

On a way to the final result we estimate the heat peak value at the figure $7[7]$ as $\left[G_{v 0}\right]^{1 / 2}$ $\cong 1,5 \cdot 10^{-1} \mathrm{~Hz} / \mathrm{Hz}^{1 / 2}$ which defines to $\left(G_{B O}\right)^{1 / 2} \cong 1,0 \cdot 10^{-13} \mathrm{~cm} / \mathrm{Hz}^{1 / 2}$. For "background" we can see $\left(G_{\vee S}\right)^{1 / 2} \cong 5 \cdot 10^{-3} \mathrm{~Hz} / \mathrm{Hz}^{1 / 2}$ or $\left(G_{S}\right)^{1 / 2} \cong 3,5 \cdot 10^{-15} \mathrm{~cm} / \mathrm{Hz}^{1 / 2}$. Then the expression (13) gives $\mathrm{A}_{\mathrm{S}} \cong$ 30. For values $f_{\mu}=1320 \mathrm{~Hz}, Q=10^{5}$ we have $\Delta f_{0}=0.013 \mathrm{~Hz}$ and find $\Delta f_{B} \cong 0,4 \mathrm{~Hz}$.

For GW detector "Ulitka" one can see $\mathrm{As}^{2} \cong 100$ on the fig. 1 and can find $\Delta f_{B} \cong 0,43 \mathrm{~Hz}$ [9]. 
The metric potential sensitivity EPD $G_{h 0}$ of installation is presented in ref. [7] and [8] at figures 8 and 5. It is obtained by recalculation of the spectrograms 7 and 4, accordingly. The expressions (10) and (11) are assumed to be used there. One can define analytical and digital dependence between values $G_{B 0}$ and $G_{h 0}$. For the amplitudes of the harmonic signal in resonance the expression (10) establish the connection: $x_{m}=h_{m} Q(L / 2)$. As above, we find the relationship

$$
\left(G_{h 0}\right)^{1 / 2}=\left(G_{B 0}\right)^{1 / 2}(2 / L) / Q
$$

For above value $G_{B O}$ we find value $\left(G_{h B}\right)^{1 / 2}=1 \cdot 10^{-20} \mathrm{~Hz}^{-1 / 2}$; it coincides with the estimate (1).

Ref. [7, 8] report that there is the "bar-mirror" displacement reduction coefficient of 1,7 . The last estimation supports the version that this coefficient is equal to one satisfactorily.

In whole, improvement in meter resolution leads to increase in detection bandwidth.

\section{Displacement transformation by mirror fastening elements}

The electrostatic method of forming calibrated forces and displacements was used before to determine the actual resolution of the measurer [11-13, 22]. This method is mentioned in the ref. [17], which provides the accuracy of 30\%, adopted in GW experiments. In resonant GWD such calibration is based on the expression (6). Registration of a thermal peak in the spectrogram provided an opportunity to create and apply an alternative additional method for determining the achieved displacement meter resolution [18].

The example of successful metrological application of the new method provides the detector "Ulitka" $[9,10]$. For values $\Delta f_{0} \cong 0,043 \mathrm{~Hz}$ and $f_{\mu}=1600 \mathrm{~Hz}$ [9] we find $Q \cong 3,8 \cdot 10^{4}$. When $\mathrm{M} \cong 500 \mathrm{~kg}$ we get: $G_{B 0} \cong 1 \cdot 10^{-13} \mathrm{~cm} / \mathrm{Hz}^{1 / 2}(9)$. For the value $\mathrm{As}_{\mathrm{S}}=10$ from the relation (14) the registration system resolution $\left(G_{S}\right)^{1 / 2} \cong 10^{-14} \mathrm{~cm} / \mathrm{Hz}^{1 / 2}$ is determined. Just this value is presented in the text [9]. Similarly, it can be an attempt to define the resolution of a laser measurer OGRAN. For values of the parameters mentioned above, we find the predicted value of $G_{B 0} \cong 1,7 \cdot 10^{-13}$ $\mathrm{cm} / \mathrm{Hz}^{1 / 2}$. For the presented value $\mathrm{A}_{S}=30$ is determines resolution $\left(G_{S}\right)^{1 / 2} \cong 6 \cdot 10^{-15} \mathrm{~cm} / \mathrm{Hz}^{1 / 2}$. Just the same value was declared before for frequency $1 \mathrm{kHz}$ without justification [16].

The left scale of the spectrograms in the figures $[5,7,8,17]$ is defined by means of the "optical calibration" Using relation (15) the calibration allows measuring the absolute values of the heat noise in displacements. As above, the peak level is observed more exactly as $G_{B 0} \cong$ 
$(0,85 \div 0,9) \cdot 10^{-13} \mathrm{~cm} / \mathrm{Hz}^{1 / 2}$, which is 2 times $(6 \mathrm{~dB})$ less than the above theoretical prediction. The discrepancy may be explained by the reducing effect of mirror oscillation amplitude at about 1.7 times compared amplitude of the bar end face due to insufficient rigidity of the fastening elements $[6,7]$, whereas the recalculation of the right spectrogram scale is not made.

Prior information about parameters of mirrors fastening oscillators can be obtained from wideband spectrograms without the special dynamic test. So, it is assumed that the peaks at frequencies below $1 \mathrm{kHz}$ are associated with the attachment of mirrors [7, 8]. The most notable are two peaks with frequencies of about 890 and $920 \mathrm{~Hz}$.

To define the transformation of displacements by these oscillators the equation of motion for a mirror oscillator outside a neighborhood of its resonance can be written

$$
m_{M} \ddot{X}_{M}+k_{M}\left(X_{M}-X_{G}\right)=0 .
$$

Here, $m_{M}$ and $k_{M}$ are the effective mass and stiffness of an oscillator, $x_{M}(t)$ and $x_{G}(t)$ are oscillatory displacements of the mirror and the bar end face.

When $x_{G}=x_{G m} \exp \left(j \omega_{\mu} t\right)$ we find the complex mirror oscillation amplitude $x_{M m}$

$$
x_{M m}=-x_{G m} /\left[\left(\omega_{\mu} / \omega_{M}\right)^{2}-1\right]
$$

Substituting the frequency values $\omega_{\mu}=2 \pi \cdot 1320 \mathrm{sec}^{-1}$ and $\omega_{M}=\left(k_{M} / m_{M}\right)^{1 / 2} \cong 2 \pi \cdot 900 \mathrm{sec}^{-1}$, we find the ratio $\omega_{\mu} / \omega_{M} \cong 1.4$ and from the expression (17) find and $x_{M m} \cong-x_{G m}$. For this case the transmission coefficient is unit. However, the model representation used is too simplified for this allocated system, and amplitude reduction is possible. Overall, the uncertainty in a range of two times may be allowed for the first OGRAN thermal noise absolute measurement and when the transfer from scientific free style of development to austere technical is carrying out.

The expression (17) excites interest to some effects. First, the change of sign means that oscillations of a mirror and an end face take place in antiphase, i.e., the attachment is not quite rigid. Also the desire to increase the rigidity $k_{M}$ leads to growth of mirror oscillation amplitude and to a resonance operation mode.

The greatest effect of oscillations increasing provides the displacement transformation at the resonant frequency $\omega_{\mathrm{M}}=\omega_{\mu}$. This mode reduces the resolution requirements to registration 
scheme. This concept is well known [23, 14, 15]; in particular, it has been realized in ref. [4]. The resonant displacement transformer is proved to be non-use at room temperature [24].

The quasi-resonant mode arises here quite naturally. The thermal peak growth at the background $G_{S}(f)$ leads to increasing of bandwidth $\Delta f_{B}$. The increase of at least a few times can replace extremely time-consuming and expensive corresponding development of the laser registration system. However, the dynamic system becomes complicated; an additional intermediate link appears; it can add thermal noise. Viscosity energy losses in the oscillators are not significantly affect the bar quality factor $Q$. After mounting of mirrors with fastening elements it decreased reasonably from a value of $Q \cong 1,6 \cdot 10^{5}[24]$ to the value of $Q \cong 10^{5}[7,8]$.

Thermal noise $G_{B M}(\omega)$ of an additional oscillator at the inlet of the displacement meter is

$$
G_{X}(\omega)=G_{X B}(\omega) / Z^{2}+G_{S}(\omega)+2 G_{B M}(\omega)
$$

where $Z\left(\omega_{M}\right)=\left(\omega_{\mu} / \omega_{M}\right)^{2}-1$ is a transformation factor.

From the equation $m_{M} \ddot{x}_{M}+H_{M} \dot{x}_{M}+k_{M} x_{M}=F_{B M}$ we can define the EPD expression for the additional thermal oscillations away from the resonance $(\omega=2 \pi f)$

$$
G_{B M}(f)=\frac{4 k_{B} T H_{M}}{k_{M}^{2}\left(1-\omega^{2} / \omega_{M}^{2}\right)^{2}}=\frac{4 k_{B} T}{m_{M} Q_{M} \omega_{M}^{3}\left(1-\omega^{2} / \omega_{M}^{2}\right)^{2}} .
$$

In the immediate vicinity of the bar frequency $\omega \approx \omega_{\mu}$ we have $G_{B M}\left(f_{\mu}\right)=G_{B M}(0) / Z^{2}$. Here $G_{B M}(0)=4 k_{B} T / m_{M} Q_{M} \omega_{\mathrm{M}}{ }^{3}$ is the oscillator thermal EPD in the quasi-static mode, mode of a gravimeter or ordinary accelerometer. In this mode calibration plates was used, when testing a displacement meters without a bar [12, 22].

For the considered oscillations mode $\mathrm{Z}<1$, assume that the meter noise in the expression (18) is small enough: $G_{S}$ « $G_{B M}\left(f_{\mu}\right)$. Then $G_{X}(\omega)=\mathrm{G}_{X B}(\omega) / Z^{2}+2 G_{B M}(0) / Z^{2}$ and in the area of thermal peak we have $A_{S}=G_{B O} / 2 G_{B M}(0)$. The background level and the potential sensitivity bandwidth are determined by the new noise source and The increase of the amplification factor $\mathrm{Z}^{-}$ ${ }^{1}$ is not useful. This effect, nevertheless, is practically useful in the case $G_{S} \geq G_{B M}(0)$.

To undertake the appropriate numerical estimates, it is necessary to assess the values $m_{M}$ and $Q_{M}$. The mass of the rod-channel with fixed mirror is about $1.3 \mathrm{~kg}$, that is, we can put $m_{M} \approx$ 
$300 \mathrm{~g}$. Of the figures in ref. $[5,7,8]$ one can give a rough estimate of $Q_{M} \approx 300$. Then $\left[2 G_{B M}(0)\right]^{1 / 2}$ $\approx 5 \cdot 10^{-15} \mathrm{~cm} / \mathrm{Hz}^{1 / 2}$, which corresponds to the background level on the spectrograms.

However, this estimate cannot provide satisfactory degree of clarity. So, if we substitute the parameters in the formula for resonance $G_{B M}\left(f_{M}\right)=4 k_{B} T Q_{M} /\left(m_{M} \omega_{M}{ }^{3}\right)$, we can obtain the value of $\left[G_{B M}\left(f_{M}\right)\right]^{1 / 2} \approx 10^{-12} \mathrm{~cm} / \mathrm{Hz}^{1 / 2}$. But the peaks in figure 2 [5] does not exceed the value of $2,5 \cdot 10^{-}$ ${ }^{1} \mathrm{~Hz} / \mathrm{Hz}^{1 / 2}$, which corresponds to $\left[G_{B M}\left(f_{M}\right)\right]^{1 / 2} \cong 1,8 \cdot 10^{-13} \mathrm{~cm} / \mathrm{Hz}^{1 / 2}$, i.e., there is the discrepancy with the calculation five times. Apparently, the model performance using simple oscillators is too simplified. Identification of the peaks in the noise spectrum, dynamic and fluctuation parameters of fastening elements require a further study. The significance of the background thermal noise of several oscillators is also noticed in the report [5].

Not-rigid mirror mounts complicate the introduction of the new heat peak calibration.

Ref. [6] contains explanation of why the bar thermal peak is not visible in the pilot model operation; the prior resolution value of the meter [17] is replaced by the value $4 \cdot 10^{-16} \mathrm{~m} / \mathrm{Hz}^{1 / 2}$. Then the peak proves to be at the threshold of detection. "Optical calibration" allows such correction. The absence of oscillator mirror attachment peaks needs to be explained too.

\section{Instrumental sensitivity}

The instrumental sensitivity of a device is determined by technical noise sources. Consider it, as above, in its phenomenological aspect. In the figures 7 and $4[7,8]$ one have a broad frequency region corresponding to the background. It is determined by the condition $G_{X B} \leq G_{S}$. Here the condition $|\Delta f|^{2} \gg\left(\Delta f_{0} / 2\right)^{2}$ is satisfied, and from the expressions (8), (10) and (11) the analytical dependence of the two linear "wings" follows

$$
\left(G_{h}\right)^{1 / 2}=\left(G_{S}\right)^{1 / 2}(2 / L Q)\left(2|\Delta f| / \Delta f_{0}\right)
$$

In fig. 8 [6] the flat section with width $\Delta f_{B}=0,4 \mathrm{~Hz}$ is not visible in noise; there the metric potential sensitivity of $\left(G_{h 0}\right)^{1 / 2}=10^{-20} \mathrm{~Hz}^{-1 / 2}$ represented by estimate (1) should be realized.

A new detector characteristic is introduced [7, 8]. It is a bandwidth at the control level of $\left(G_{h c}\right)^{1 / 2}=10^{-19} \mathrm{~Hz}^{-1 / 2}$. This band is determined from the dependence $\left[G_{h}(f)\right]^{1 / 2}(19)$. Thus, there are two values of frequency $f$ in which the condition $\left[G_{h}(f)\right]^{1 / 2}=\left(G_{h c}\right)^{1 / 2}$ is satisfied. Due to the linearity of the dependence the difference of these two frequencies $\Delta f_{G}$ is given by relation 


$$
\Delta f_{G}=\Delta f_{B}\left[G_{h c}(f)\right]^{1 / 2} /\left[G_{h 0}(f)\right]^{1 / 2} \equiv B_{h} \Delta f_{B}
$$

Here for $B_{h}=10$ and resulting value is " $\Delta f_{G}=4 \mathrm{~Hz}$ " [7, 8] is obtained. So one can understand the meaningful of the introduced enhanced receive bandwidth. It follows directly from the peak and background values shown in figure 7 [7] (fig.4 [8]). Those steps complete the phenomenological algorithm for transformation of displacement noise spectrogram.

The rigorous analysis the GW detector sensitivity is presented by the expressions (1). This premise indicates the continuity and priority of MSU in the collaboration. The noise factor $F$ is presented by formula [15]: $F=(2 M / \tau)\left[G_{V}(\omega) / G_{B}(\omega)\right]^{1 / 2}$.

The value of $G_{V}(\omega)$ characterizes the certain laser registration scheme $[2,14,18]$

$$
G_{V}(\omega)=B \omega_{\mu}^{2}(2 h v / \eta W)(\lambda / 2 \pi N)
$$

where $\omega_{\mathrm{e}}, \lambda$ and $W$ are the frequency, wavelength and laser power, $\eta$ is the photodiode quantum efficiency, $N$ is "the number of reflections" and $B$ is "a multiplier that takes into account the excess noise real laser above the level of the shot noise" [17]. A gravitational wave is expected in the quasi-harmonic form $F_{S}(t)=F_{0}(t) \sin \omega_{\mu} t$ with burst duration $\tau \approx(3 \div 10) \cdot 2 \pi / \omega_{\mu}[15]$.

Sensitivity becomes instrumental when $\mathrm{F}>1$. The estimates $F \approx 1$ are available in ref. [4, 17]. This should mean the instrument is a ready detector for an expected signal exposure. Also the phenomenon of interest is the calculated value $F<1$, which is easy to obtain bellow for real parameter values. While considering about the factor $F$ it is advisable to follow the option (21).

For the pilot model parameter values $L=0,5 \mathrm{~m}: M=25 \mathrm{~kg}, \omega_{\mu}=2 \pi \cdot 5 \cdot 10^{3} \mathrm{sec}^{-1}, Q=$ $1,7 \cdot 10^{4}[17]$ the expression (3) give $\mathrm{G}_{B}(\omega) \cong 1,2 \cdot 10^{-19} \mathrm{~N}^{2} \mathrm{sec}$. For values $\lambda=1,06 \cdot 10^{-6} \mathrm{~m}, \eta=0,6$, $W=1 \mathrm{~W}, B=1, N=10^{3}$ the expression (21) gives $G_{V}(\omega) \cong 1,8 \cdot 10^{-29} \mathrm{~m}^{2} / \mathrm{sec}^{3}$. For pulse duration $\tau=3 / f_{\mu}=6 \cdot 10^{-3}$ sec one obtain $F \cong 1$. When $\tau=6,3 / f_{\mu}[17]$ one have $F \cong 0,5$.

For full-scale detector for the values $L=2 \mathrm{~m}, M=10^{3} \mathrm{~kg}[24], \omega_{\mu}=2 \pi \cdot 1,3 \cdot 10^{3} \mathrm{~s}^{-1}, Q=10^{5}$ $[17,7,8]$ the estimate $\mathrm{G}_{B}(\omega) \cong 2,2 \cdot 10^{-19} \mathrm{~N}^{2}$ sec follows. For values $\eta=0,6, W=1 \mathrm{~W}, B=1, N=$ $1,5 \cdot 10^{3}$ [4] one findes $G_{V}(\omega) \cong 5,5 \cdot 10^{-31} \mathrm{~m}^{2} / \mathrm{sec}^{3}$. When $\tau=4 / f_{\mu}=3 \cdot 10^{-3} \mathrm{sec}$ one can find $F=1,06$ $\approx 1$. When $\tau=8 / f_{\mu}$ one finds $F=0,53$. For $\tau=0,25 \mathrm{sec}$ we find $F=0,013[7,8]$.

To clarify the noise factor expression, consider its derivation [15]. The previous expression is: $F \cong(\delta \tau)^{-1}$, where $\delta^{2} \cong(2 M)^{-2} \mathrm{G}_{B} / G_{V}$. It has been derived for the case $\delta \tau \ll 1$, i.e., for $F » 1$. Since then technology development has required expansion of analysis to the area of moderate and small 
values of $F<10$. The base expansion has the view: $F_{c}=[\delta \tau \cdot \arctan (1 / \delta \tau)]^{-1}$. At the case $\delta \tau \ll 1$ one have $F_{c}=(2 / \pi)(\delta \tau)^{-1}$. In the opposite case $\delta \tau » 1$ one have $F_{c} \cong 1$; the revision eliminates the mode $\mathrm{F} \leq 1$. In the intermediate value aria as a boundary we define $F_{c}=2$, i.e. $3 \mathrm{~dB}$. The boundary duration is $\tau_{0} \cong 0,43 \delta^{-1}$. For above parameter values we have $\delta \cong 3 \cdot 10^{2} \mathrm{sec}^{-1}$ and $\tau_{0} \cong 1,4 \cdot 10^{-3} \sec$ $\approx 2 / f_{\mu}$. It defines wide detector receive frequency band $1 / \tau_{0} \cong 700 \mathrm{~Hz}$.

Consider a phenomenon of $F \approx 1$ in calculations. The analytical expression for resolution of the laser measurer is showed [18]: $G_{S O}(\omega)=2 B(\lambda / 2 \pi N)^{2} h v / \eta \mathrm{W}$. We calculate $G_{S O}(\omega)=0,8^{-1} 10^{-}$ ${ }^{38} \mathrm{~m}^{2} \mathrm{sec}$ or $\left[G_{S O}(f)\right]^{1 / 2}=2,3 \cdot 10^{-17} \mathrm{~cm} / \mathrm{Hz}^{1 / 2}$. From the expressions $(13),(14)$ for $\left(G_{B O}\right)^{1 / 2}=1,7 \cdot 10^{-}$ ${ }^{13} \mathrm{~m} / \mathrm{Hz}^{1 / 2}$ we find $\mathrm{As}_{\mathrm{S}}=7,5 \cdot 10^{3}$ and $\Delta f_{\mathrm{B}} \cong 100 \mathrm{~Hz}$. Thus, value of $F \approx 1$ means the achievement the theoretical limit resolution of the displacement measuring circuit [2]. This example also may be assumed as the first step towards the narrowing of the gap between the phenomenological and analytic algorithms for calculation of resonant detector potential sensitivity bandwidth.

In the OGRAN detector is implemented complicated displacement meter optoelectronic scheme in PDH technique with internal modulation, synchronous demodulation and using reference FP cavity discriminator of FM laser radiation [4]. The use of another, conceptual measuring circuit $[2,14]$ for sensitivity prediction is a demonstration of a liberal scientific style. In ref. [18] a general analysis of PDH scheme is presented; the basic shot noise of photoelectrons was considered. Also the excess technical noise source of stochastic variations of the real laser radiation power envelope was allowed for; ILP entered its contribution by a suitable factor $B_{H}, B_{H}$ $\approx 1,6(\sim 2 \mathrm{~dB})$ at the modulation frequency of $10.7 \mathrm{MHz}$.

If mirrors of each cavity have the same optical parameters and values of the radiation power $P_{P H}$ falling on the photodiodes are equal, the PDH meter resolution is predicted by formula [18]

$$
G_{S}(f) @ B_{H} \lambda^{2} h n P_{P H}\left(32 h P_{C} P_{S}\right)^{-1}\left[\left(K_{G} F_{G}\right)^{-2}+\left(L / L_{D}\right)^{2}\left(K_{D} F_{D}\right)^{-2}\right]
$$

Here $L_{D}$ is discriminator reference bar length, $\Phi_{G, D}$ is a finesse of FP cavity, $P_{P H}$ is power of IR light at the photodiode surface, $P_{C}$ and $P_{S}$ are the carrier and sideband power components, $K_{G, D}$ is a FP cavity contrast, introduced into consideration [16]: $P_{P H}=\left(1-K_{G, D}\right) P_{C}+2 P_{S}[20]$.

The relation $G_{V}(\omega)=\omega_{\mu}{ }^{2} G_{S O}(\omega)$ is determined [18]. When using expression (22) we have $G_{V(P D H)}(\omega)=B_{H} \omega_{\mu}{ }^{2} \lambda^{2} h v P_{P H}\left(64 \pi \eta P_{C} P_{S}\right)^{-1}\left[\left(K_{G} \Phi_{G}\right)^{-2}+\left(L / L_{D}\right)^{2}\left(K_{D} \Phi_{D}\right)^{-2}\right]$. 


\section{Noise estimates}

The bar thermal peak is visible on two spectrograms [7] ([8]). At broadband one at the fig. 6 we can see $\left(G_{B 0}\right)^{1 / 2} \cong 2 \cdot 10^{-13} \mathrm{~cm} / \mathrm{Hz}^{1 / 2}$. It corresponds kindly to the estimate of $1,7 \cdot 10^{-13} \mathrm{~cm} / \mathrm{Hz}^{1 / 2}$, obtained using the expression (9). As noted above, on the spectrograms with higher frequency resolution (fig. 4,7 ) the peak value is $\left(G_{B 0}\right)^{1 / 2} \cong 10^{-13} \mathrm{~cm} / \mathrm{Hz}^{1 / 2}$. The discrepancy is twice

The potential sensitivity of the detector is determined by the expression (1) [7]; it is very approximate due to complexity of the transition from the pulse duration $\tau$ to the metric EPD. Consequently the estimates of $\left(G_{\mathrm{h} 0}\right)^{1 / 2} \approx 1 \cdot 10^{-20} \mathrm{~Hz}^{-1 / 2}$ and the resulting bandwidth of $\Delta f_{G} \approx 4 \mathrm{~Hz}$ are approximate too. Meanwhile, a more accurate expression (5) is derived by direct. For $Q=10^{5}$ $[6,7]$ the value $\left(G_{\mathrm{h} 0}\right)^{1 / 2}=1,7 \cdot 10^{-20} \mathrm{~Hz}^{-1 / 2}$ follows it from. The value $\left(\mathrm{G}_{\mathrm{h} 0}\right)^{1 / 2}=1,5 \cdot 10^{-20} \Gamma_{\bigsqcup^{-1 / 2}}$ was obtained from the formula (1) for $Q=1,6 \cdot 10^{5}$ [24]. If in the expression (16) as above use the calculated value of $\left(G_{B 0}\right)^{1 / 2} \cong 1,7 \cdot 10^{-13} \mathrm{~cm} / \mathrm{Hz}^{1 / 2}$, we get more precise value $\left(G_{\mathrm{h} 0}\right)^{1 / 2}=1,7 \cdot 10^{-20} \mathrm{~Hz}^{-}$ ${ }^{1 / 2}$. While using the expression (22) we get $B_{h} \cong 6$ and the specified value $\Delta f_{G c} \cong 2,4 \mathrm{~Hz}$.

For the detector "Ulitka" $(M \cong 500 \mathrm{~kg}[10])$ we obtain similarly $\left(G_{h 0}\right)^{1 / 2}=3,8 \cdot 10^{-20} \mathrm{~Hz}^{-1 / 2}$ and $B_{h}=2,6$; for $\Delta f_{B}=0,43 \mathrm{~Hz}$ we find $\Delta f_{G}=1,1 \mathrm{~Hz}$. These values are presented at the Table 2 .

Comparison of values of $2,4 \mathrm{~Hz}$ and $1,1 \mathrm{~Hz}$ indicates insignificant difference of OGRAN setup and detector "Ulitka". However, the better result can be found by careful study. Thus, in fig. 6 [7] and fig. 3 [8] the thermal peak level corresponds well to calculated value of $1,7 \cdot 10^{-13}$ $\mathrm{cm} / \mathrm{Hz}^{1 / 2}$, whereas the pointed out background level is $\left(G_{S}\right)^{1 / 2} \cong 2 \cdot 10^{-15} \mathrm{~cm} / \mathrm{Hz}^{1 / 2}$ [6]. We can calculate $\mathrm{A}_{S}=85, \Delta f_{B}=1,1 \mathrm{~Hz}$ and $\Delta f_{G c} \cong 6,5 \mathrm{~Hz}$. This gain is a consequence of the thermal peak height doubling in comparison with the next figure. The main result $\left(\Delta f_{G c} \cong 4 \mathrm{~Hz}\right)[7,8]$ is preferable because value $A_{S}=85 \div 100$ is not saved while increasing the resolution of spectrum analysis; it is closer to a real, completed instrument. Another reason could be that the most modest result in metric should suit to the beginning of the transition period in BNO.

A similar calculation of a new receive bandwidth can be performed for value of $Q=5000$. From the expressions (9) and (13), we obtain $\left(G_{B 0}\right)^{1 / 2}=3,8 \cdot 10^{-14} \mathrm{~cm} / \mathrm{Hz}^{1 / 2}$ and $\mathrm{As}_{\mathrm{S}}=19$. Next, the following values are calculated: $\Delta f_{0}=0,26 \mathrm{~Hz}, \Delta f_{B}=4,95 \mathrm{~Hz},\left(G_{\mathrm{h} 0}\right)^{1 / 2}=6,7 \cdot 10^{-20} \mathrm{~Hz}, B_{h}=1,3$ and $\Delta f_{G}=6,5 \mathrm{~Hz}$. This calculation show clearly that the bandwidth has not changed. The formulas presented allow us to show analytically that the dependence on quality factor $Q$ is excluded. This new effect provides an opportunity for revision of acoustic resonator design to create GW detectors at substantially lower frequency with moderate length.

The value of $\left(G_{S} f\right)^{1 / 2} \cong 3 \cdot 10^{-16} \mathrm{~cm} / \mathrm{Hz}^{1 / 2}$ is presented [7]. Its connection with figure 7 is not clear, it looks like a secondary independent result. The figure can be perceived as a result of 
progressive improvement of the displacement meter. So, we have a series: $6 U$ [17], 4U [5], 2U [6], $0,6 \mathrm{U}[8]$ and $0,3 \mathrm{U}$, where $\mathrm{U}=10^{-15} \mathrm{~cm} / \mathrm{Hz}^{1 / 2}$. The last quantity is especially valuable if it is referred out of the context. The relevant calculation can be performed. Assuming $\left(G_{B 0}\right)^{1 / 2} \cong 1,7 \cdot 10^{-}$ ${ }^{13} \mathrm{~cm} / \mathrm{Hz}^{-1 / 2}$, from (13) we find formally $\mathrm{A}_{S} \cong 565$. Then, from the expression (14) we find $\Delta f_{B} \approx$ 7,5 Hz. For optimistic estimate (1), we find quite wide forecast receive band $\Delta f_{G} \approx 75 \mathrm{~Hz}$.

Table 1. The parameter values and resulting metric characteristics are summarized. Consider contributions of the important noise sources in the displacement meter.

\begin{tabular}{|c|c|c|c|c|c|c|}
\hline & $\begin{array}{c}\left(G_{B O}\right)^{1 / 2}, 10^{-13} \\
\mathrm{~cm} / \Gamma^{-1 / 2}\end{array}$ & $\begin{array}{c}\left(G_{S}\right)^{1 / 2} \cdot 10^{-15} \\
\mathrm{~cm} / \Gamma^{1 / 2}\end{array}$ & $\mathrm{~A}_{S}$ & $\begin{array}{c}\Delta f_{B}, \\
\Gamma_{ц}\end{array}$ & $\begin{array}{c}\Delta f_{G}, \\
\Gamma_{ц}\end{array}$ & $\begin{array}{c}\Delta f_{G c}, \\
\Gamma ц\end{array}$ \\
\hline "Ulitka" [9], fig. 1 & 1 & 10 & 10 & 0,43 & - & 1,1 \\
\hline Fig. 7, 4 [7, 8] & 0,9 & 3 & 30 & 0,4 & 4 & 2,4 \\
\hline Fig. 2 [5], & 1,2 & 4 & 30 & 0,4 & 4 & 2,4 \\
\hline Text [6] & 1,7 & 2 & 85 & 1,1 & 11 & 6,5 \\
\hline Fig. 3, 6 [7, 8], & 2 & 2 & 100 & 1,3 & 13 & 7,5 \\
\hline Text to fig. 4 [8], & 1,7 & 0,6 & 285 & 3,7 & 37 & 21 \\
\hline Text to fig. 7 [7], & 1,7 & 0,3 & 565 & 7,5 & 75 & 43 \\
\hline Designed [4] & 1,7 & 0,1 & 1700 & 22 & 220 & 130 \\
\hline
\end{tabular}

Laser radiation provides the main noise source. In the expression (21) it is characterized by factor $B \approx 10^{4}[13]$ or $B \approx 1 \div 10^{3}[7,8]$. This factor reduces substantially technical value of the calculation, whereas scientific one beyond doubt. This low frequency (LF) source consists of laser power fluctuation (LPF) component and component of laser frequency fluctuations (LFF). The PDH technique excludes the LF LPF component; it is replaced by high frequency (HF) LPF with reasonable factor $B_{H}$. Meanwhile, LF LFF inherent component remains in full [18]. In ref. [17] at the LFF spectrum one can see $\left(S_{v 0}\right)^{1 / 2} \cong 4,5 \mathrm{~Hz} / \mathrm{Hz}^{1 / 2}$ for frequency of $1,3 \mathrm{kHz}$. In ref. [25] it was assayed as $\sim 10 \mathrm{~Hz} / \mathrm{Hz}^{1 / 2}$; dependence on laser bias mode, time and others is implied.

Suppression of LFF is performed by stabilization using a reference cavity. In the OGRAN project the bar FP cavity executes this function itself. Taking into account uniqueness of the task a special justification was made. In conjunction with other noise sources an evident expression for the laser frequency EPD $S_{v}$ in locked mode was derived: $S_{v}=S_{v 0} /\left|K_{0}\right|^{2}[26,25]$. Here $K_{0}$ is the feedback gain at the frequency 1,3 kHz. Gain enlargement had required the engineering of highspeed laser frequency tune circuit, including introduction the third driver channel with EOM [16]. 
The value $K_{0} \cong 10^{3}$ is implemented $[7,8,17]$. Then we get the estimate $S_{v} \cong(5 \div 10) \cdot 10^{-3} \mathrm{~Hz} / \mathrm{Hz}^{-1 / 2}$ or $\left(G_{S}\right)^{1 / 2} \cong(3 \div 7) \cdot 10^{-15} \mathrm{~cm} / \mathrm{Hz}^{-1 / 2}$; it is close to "background" at the spectrum figures.

The base photoelectron shot noise defines the limit resolution of a PDH displacement meter. In ref. [7] all parameter values contained in the formula (24) are presented. The phase modulation index 1,15 rad determine the ratio $P_{S} \cong 0,4 P_{C}$. For value $P_{P H 1} \cong 50 \mathrm{~mW}$ we find $P_{C}=$ $30 \mathrm{~mW}$ and $P_{S}=12 \mathrm{~mW}$. Substituting the values $L_{D}=0,45 \mathrm{~m}, \Phi_{G}=3000, \Phi_{D}=12000, K_{G}=0,2$, $K_{D}=0,4, \eta=0,7$ we get the estimate: $\left(G_{S 2}\right)^{1 / 2} \cong 3 \cdot 10^{-16} \mathrm{~cm} / \mathrm{Hz}^{1 / 2}$. The first term is dominated; the discriminator channel introduces the acceptable addition of $15 \%$. In ref. $[4,17,26]$ resolution of a displacement meter is considered under the assumption that a discriminator is designed correctly, that is, its noise contribution is negligible. It is presented here.

A RAM noise is mentioned as a dominant [6 - 8]; no calculation has not presented. Residual radiation modulation depth $m_{R}$ generates the photodiode AC component; its amplitude is $I_{0} m_{R}$, where $I_{0}=(\eta e / h v) P_{P H}$ is the DC. The corresponding addition of the synchronous detector output voltage carries LPF. The reduced stochastic current have EPD $S_{I R}=S_{m N} I_{0}^{2} m_{R}^{2}$, where $S_{m N}$ is a normalized EPD of LF LPF [20]. Displacement resolution is defined by a general expression: $\left(G_{S}\right)^{1 / 2}=\left(S_{I R}\right)^{1 / 2} / \alpha_{x I}$, where $\alpha_{x I}$ is a parameter of "displacement-photocurrent" conversion:

$$
\alpha_{x I}=d I / x=8 F_{G} K_{G}(h e / h n)\left(P_{C} P_{S}\right)^{1 / 2} / I
$$

For a real FP cavity the formula needs experimental verification and correction. For above parameter values [7] we get estimates: $\alpha_{x I} \cong 5 \cdot 10^{7} \mathrm{~A} / \mathrm{m}, I_{0}=30 \mathrm{~mA}$. In the absence of data for calculation we have to use the value $S_{m N}=10^{-12} \mathrm{~Hz}^{-1}[20]$ and to match the parameter $m_{R}=0,5$ to obtain the presented value $\left(G_{S}\right)^{1 / 2} \cong 3 \cdot 10^{-16} \mathrm{~cm} / \mathrm{Hz}^{1 / 2}$ as an estimate.

The articles [7], [8] contain a lot in common. Substantial differences take place. The other values of parameters are presented: $\Phi_{D}=15000, K_{G}=0,3, K_{D}=0,2$ [8]. There pointed out that the laser power falling to the cavities is $50 \mathrm{~mW}$ and also $P_{P H} \cong 10 \mathrm{~mW}$. We find $P_{C} \cong 6 \mathrm{~mW}$ and $P_{S} \cong 2,5 \mathrm{~mW}$. Then the estimate of shot noise meter resolution is $6 \cdot 10^{-16} \mathrm{~cm} / \mathrm{Hz}^{1 / 2}$. It coincides with the announced figure. In this embodiment the discriminator noise contribution is relatively high. The RAM noise in it is noticeable too. Ref. [6] indicates that RAM is formed at photodiode surfaces. It means the RAM suppression must be carried out in both optoelectronic channels. 


\section{Conclusion}

Non rigid FP mirror mounts provide a notable additional thermal noise into GW detector.

In addition to reported result in the OGRAN full-scale installation metric sensitivity the independent figure $(3 \div 6) \cdot 10^{-16} \mathrm{~cm} / \mathrm{Hz}^{1 / 2}$ is revealed. While interpreting it as a result of tests, the figure means an outstanding improvement in resolution of the laser displacement meter as a main achievement of the Moscow period of the Project development.

If applying the phenomenological displacement transformation algorithm to this advanced figure, calculation leads to a significant improvement of resulting characteristic of the OGRAN installation in gravitational field metric; it suits to the end of the transition period in BNO.

Calculation shows that the presented figure closely corresponds to the theoretical prediction of displacement meter resolution defined by the photoelectron shot noise. Account of the laser radiation technical noise projects the increase of the displacement meter threshold signal.

\section{References}

1. Weber J. (1966). Observation of the thermal fluctuations of a gravitational-wave detector. Phys. Rev. Lett., Vol. 17, 1228-1230.

2. Braginsky V.B. (1970). Physical Experiments with Test Bodies. Moscow: Nauka [Science].

3. Conti L., Cerdonio M., Taffarello L., Zendri J.P., Ortolan A., Rizzo C., Ruoso G., Prodi G.A., Vitale S., Cantatore G., Zavattini E. (1992). Optical transduction chain for gravitational wave bar detectors. Review of Scientific Instruments, Vol. 69, No.2, 554-558.

4. Bezrukov L., Popov S., Rudenko V., Serdobolskii A., Skvortsov M. (2004). Gravitational wave experiments and Baksan project "OGRAN". Proc. Int. Conf. "Astrophysics \& Cosmology after Gamow”, 19.

5. Bezrukov L.B., Kvashnin N.L., Motylev A.N., Oreshkin S.I., Popov S.M., Rudenko V.N., Samoilenko A.A., Skvortsov M.N., Yudin I.S., (2013). New opto-acoustical gravitational detector in BNO INR RAS. Proc. Int. Meeting “PIRT-2013”, Moscow: BMSTU, 23-29.

6. Vishnyakov V.I., Ignatovich S.M., Kvashnin N.L., Popov S.M., Rudenko V.N., Samoilenko A.A., Skvortsov M.N., Yudin I.S. (2013). Suppression of residual amplitude modulation of electro-optical modulator in OGRAN project. Tech. Digest Int. Symp. “MPLP-2013”, 179.

7. Bagaev S.N., Bezrukov L.B., Kvashnin N.L., Krysanov V.A., Motylev A.M., Oreshkin S.I., Popov S.M., Rudenko V.N., Samoilenko A.A., Skvortsov M.N., Yudin I.S. (2015). An Optoacoustical Gravitational Antenna. Instruments and Experimental Techniques, No.2, 95115 . 
8. Bagaev S. N., Bezrukov L. B., Kvashnin N. L., Krysanov V. A., Oreshkin S. I., Motylev A. M., Popov S. M., Rudenko V. N., Samoilenko A. A., Skvortsov M. N., Yudin I. S. (2014). A high frequency resonance gravity gradiometer. Review of Scientific Instruments, Vol. 85, № 6.

9. Gavrilyuk Yu M., Gusev A.V., Krysanov V.A., Kulagin V.V., Motylev A.M., Oreshkin S.I., Rudenko V.N., Silin V.A., Tsepkov A.N. (2012). Analysis of the "Ulitka" noise background as an anti-coincidence filter for the OGRAN gravitational-wave antenna. Astronomy Reports, Vol. 56, No.8, 638-652.

10. Gusev A.V., Kulagin V.V., Oreshkin S.I. Rakhmanov A.N., Rudenko V.N., Serdobol'skii A.V., Tsepkov A.N., Tsyganov A.V., Motylev A.N. (1997). Observations of the gravitational gradient background using the Ulitka GW antenna. Astronomy Reports, Vol.41. No.2. 248-256.

11. Braginsky V.B., Mitrofanov V.P., Rudenko V.N., Khorev A.A. (1979). Measurement of weak acoustic waves using capacitive sensor. Prib. Tekh. Eksp. No.4, 241-243.

12. Braginsky V.B., Panov V.A,, Petnikov V.G. and Popel'nyuk V.D. (1977). The measurement of small mechanical vibrations by means of the capacitive sensor with superconducting resonator. Prib. Tekh. Eksp., No.1, 234-236.

13. Krysanov V.A., Kuklachov M.I. and Rudenko V.N. (1979). Parametric sensor in a dynamic damping mode. Prib. Tekh. Eksp. No.4, 240-243.

14. Kulagin V.V., Polnarev A.G. and Rudenko V.N. (1986). A combined optical-acoustical gravitational antenna, Sov. Phys. JETP, Vol.64, 915-921.

15. Gusev A.V., Kulagin V.V. and Rudenko V.N. (1996). Room-temperature gravitational bardetector with cryogenic level of sensitivity. Gravitation \& Cosmology, No.5, 68-70.

16. Rudenko V.N., Popov S.M. (2007). Pilot model of opto-acoustic gravitational-wave antenna. Proc. Int. Conf “PIRT'2007”, 49-54.

17. Bezrukov L.B., Kvashnin N.L., Motylev A.M., Oreshkin S.I., Popov S.M., Rudenko V.N., Samoilenko A.A., Skvortsov M.N., Tsepkov A.N., Cheprasov S.A., Yudin I.S. (2010). A precise system for measuring weak optoacoustic perturbations. Instruments and Experimental Techniques, V. 53, No.3, 423-429.

18. Krysanov V.A. (2011). Sensitivity of laser meters of small oscillations. Vestnik MGTU. Estestvennye nauki [Herald of BMSTU. Natural science], 163-179.

19. Krysanov V.A. (2012). Signal and fluctuation characteristics of the laser registration system for GW detector. Proc. Int. Meeting “PIRT-2011”, Moscow: BMSTU, 165-177. 
20. Krysanov V.A. (2011). Influence of the optical pumping power fluctuations on the OGRAN detector sensitivity. Gravitation \& Cosmology, Vol. 17. No.1, 97-100.

21. Braginsky V.B. (1993). Detection of gravitational waves: problems and prospects. Vistas in Astronomy, Vol. 37, 341-345.

22. Krysanov V.A., Rudenko V.N. (1984). Magneto-dynamic measurer of small mechanical vibrations. Prib. Tekh. Eksp., No.3, 199-203.

23. Lavrent'ev G.Ya. (1969). Gravitational resonant detector with two degrees of freedom. JETP Letters, Vol.10, No.10, 318-321.

24 Gusev A.V., Rudenko V.N, Cheprasov S.A., Bassan M. (2008). Reception frequency bandwidth of a gravitational resonant detector with optical readout. Class. Quantum Grav, Vol.25, 055006.

25. Krysanov V.A. (2009). Suppression of laser frequency fluctuations in project "OGRAN". Proc. Int. Meeting “PIRT-2009”, Moscow: BMSTU, 242-249.

26. Krysanov V.A. (2008). Instrument sensibility of optical-electronic scheme for detection of acoustic oscillations of GW antenna. Proc. Int. Meeting "PIRT-2007”, Moscow: BMSTU, 55-61. 